\title{
Taxonomic Studies on some species of genus Huernia R.Br. (Asclepiadaceae) growing in AL-Taif Province, Saudi Arabia
}

\author{
Nahed M. Waly* and Samah A. Al-Rehaily** \\ *Faculty of Science - Botany department- Cairo University, Egypt \\ **Applied Faculty of Science, Biology Department- Umm Al-Qura \\ University, Saudi Arabia \\ *Corresponding author, e-mail: nwaly89@hotmail.com
}

Nahed M. Waly and Samah A. Al-Rehaily 2013. Taxonomic Studies on some species of genus Huernia R.Br. (Asclepiadaceae) growing in AL-Taif Province, Saudi Arabia. Taeckholmia 33: 1-18.

\begin{abstract}
Morphological characters of Huernia (Asclepiadaceae) play an important role in taxonomic identification. The present work is a revision of the taxonomic relationships based on morphological criteria of Huernia species growing in Wadi Thi-Ghazal or Al-Ghazal in the Al-Shafa region in Al-Taif province (SW Arabia). The objective was describing and scientifically authenticating the exotic species in the study area. The present revision revealed the presence of eight taxa belonging to Huernia from Asir area, three of these taxa were previously described, two are closely related to $H$. khalidbinsultanii Plowes \& McCoy, and three are unresolved taxa.
\end{abstract}

Key words: Huernia spp., Taif Province, Asclepiadaceae.

\section{Introduction}

The genus Huernia R.Br. belongs to family Asclepiadaceae and includs about 70 species, distributed in the tropical part of the world (Court, 2000). The genus is characterized by large flowers $(5-8 \mathrm{~cm}$ diam.), hairy to varying degrees, with color of mimics rotting meat and generate the odor of rotten 
flesh which attracts scavenging flies, for pollination. It is believed that the putrid smell is created, at least to a certain degree, by amine compounds (putrescine and cadaverine), which are derived from the amino acids lvsine and ornithine. (Armstrong, 1997). These amines stink and are produced when proteins rot and decompose.

Huernias are curious little plants, usually with short, fleshy, angular stems which are leafless and normally branched from the base; they are glabrous and either simply toothed or tipped with soft bristles. The campanulate flowers, which usually develop towards the base of the stem, have ten corolla lobes, consisting of five main lobes (outer corona) with sinuses extended to form five extra, inner lobes (inner corona). The inner surface of the flowers is variously and characteristically covered in papillae, presumably a modification for pollination providing obstacles to the approach or escape of certain insects.

Survey of the relevant literature Forsskål (1775), Brown (1895), Schwrtz (1939): Lavranos (1963): Field (1981), Collenette (1985, 1998; 1999), Heemstra \& Minwer (1990), Miller and Cope (1996), Migahid (1996), Wood (1997), ). Chaudhary and lawai (1999) showed that the genus Huernia is represented in the flora of the Kingdom of Saudi Arabia by eight species of which only three are named viz.: $H$. arabica, $H$. laevis and $H$. saudi-arabica. Collenette (1999) in her wild flowers of Saudi Arabia recorded another five un-named species of Huernia on the basis of her own collections. Al-Hemaid (2001) added a new species; Huernia haddaica to the genus Huernia from Saudi Arabia as an endemic species in the Al-Hada near Al-Taif which was proved to be an illegitimate and is now treated as synonym to $H$. khalidbinsultanii by Plowes \& McCoy (2003) Based on the above citation we can say that flora Saudi Arabia currently has eight species of the genus Huernia viz.

Accordingly, eight species are known in the western and southwestern regions of Saudi Arabia viz.: H. arabica D.V.Field, H. laevis JRI Wood, H. khalidbinsultanii Plowes \& D.C.H., H. saudi arabica N.E.Br. and two dubious defined taxa: Huernia sp. nov. aff. boleana, H. sp. aff. lodarensis. In addition to two unnamed Huernia sp 3610 and Huernia sp1176; the last six taxa are believed to be endemic to SW Arabia. However, Wadi AlGazal in Al-Shafa region (Al-Taif province) is the richest area with many species of the genus. 


\section{Materials and Materials}

The study area of Wadi Thi-Ghazal in the Al-Shafa region (SW Arabia $2000 \mathrm{~m}$ (AMSL) Above Medium See Level) is the richest area with vegetation in Al-Taif province, including many species of genus Huernia. Plant specimens were collected from the study area during the flowering period between March and September 2010 and the flowers were kept in a conservation solution $(2 \mathrm{ml}$ formalin, $1 \mathrm{ml}$ glycerol, $20 \mathrm{ml}$ of distilled water), according to Leach (1995) to be examined in the laboratory. Herbarium specimens of all taxa are kept in the herbarium of King AbdulAziz University (Girls section) Jeddah, Saudi Arabia. The measurement, description, and photography of the vegetative and floral plant organs were examined by the aid of a binuclear microscope; NOVEX AP-8. These include stem morphology (angles, width of tubercles) and the floral morphology (pedicel length, flower diameter, sepals and petals characters as well as corona characters). Quantitative morphological characters of recognized eight taxa are shown in tables 1 and 2. Qualitative characters include petal odor and color as well as outer and inner corona characters. All photos were taken by SONY Cyber-shot DSC-W350 camera. (Figs 2-9) Using a special program to create a dendrogram from a similarity matrix. The program calculates a similarity matrix transforms similarity coefficients into distances and makes a clustering using the Unweighted Pair Group Method with Arithmetic mean (UPGMA) algorithm.

\section{Results and Discussion}

Quantitative and qualitative morphological characters of the eight investigated species of Huernia genus are summarized in Tables 1 \& 2 Based on 26 morphological characters studied, we recognized 15 major characters to categorize the eight Huernia species under investigation. From those characters, we identified pedicle length, corolla length, and diameter, corolla tube length and diameter, corolla lobe length and width, corolla papilla color, type and length. In addition, we studied outer corona diameter, inner corona lobe, type and length, as well as odor characteristics. 
Table 1. Quantitative morphological characters of eight Huernia taxa under investigation.

\begin{tabular}{|c|c|c|c|c|c|c|c|c|}
\hline \multirow[b]{3}{*}{ se aaeaC } & \multirow{3}{*}{$\begin{array}{c}\text { fo } \quad \text { rahmY } \\
\text { stem } \\
\text { cel gaC }\end{array}$} & \multirow{3}{*}{$\begin{array}{l}\text { sra m } \\
\text { gad rr } \\
\text { a }\end{array}$} & \multicolumn{2}{|c|}{$\begin{array}{l}\text { Tubercles on stem } \\
\text { ) ude Wadngacda }\end{array}$} & \multicolumn{4}{|c|}{ Flower } \\
\hline & & & \multirow{2}{*}{\multicolumn{2}{|c|}{\begin{tabular}{|c|c|} 
edrrm mad & gaming \\
ror ahaogal & ror ahagaC
\end{tabular}}} & \multirow{2}{*}{$\begin{array}{c}\text { Pedicel } \\
\text { length } \\
\mathrm{cm}\end{array}$} & \multirow{2}{*}{\begin{tabular}{|c|} 
Flower \\
diameter \\
$\mathrm{cm}$
\end{tabular}} & \multicolumn{2}{|c|}{$\begin{array}{c}\text { Sepals } \\
\text { mm }\end{array}$} \\
\hline & & & & & & & $\mathrm{L}$ & $\mathrm{W}$ \\
\hline H.saudi arabica & 5 & $6-8$ & $3-4$ & $6-9$ & $11.5-12$ & $3.5-4$ & $1-2.5$ & $10-13$ \\
\hline H.khalidbinsultanii & 5 & $5-8$ & $2-3$ & $9-15$ & $6.5-7.5$ & $2.5-3.5$ & $1.5-2$ & $14-17$ \\
\hline Huernia .sp 1 & 5 & $16-8$ & $3-4$ & $01-14$ & $7-9.5$ & $2.5-3$ & $1-2$ & $12-17$ \\
\hline Huernia .sp 2 & 5 & $6-8$ & $2-2.5$ & $7 \mathrm{m9}$ & $21-22$ & $4.5-5$ & $1.2-1.5$ & $12-15$ \\
\hline $\begin{array}{l}\text { Huernia sp. } \\
\text { (Collenette 1176) }\end{array}$ & 5 & 1ธ. $5-6$ & $1-1.5$ & $5-6$ & $15-16.5$ & $3.5 \mathrm{~m} 5$ & $1.75-2$ & $12-14$ \\
\hline Huernia sp.3 & 5 & $6-9.5$ & $2-5$ & $11-15$ & $11.5-12$ & $3-3.3$ & $1.5-2$ & $12-13$ \\
\hline Huernia sp.4 & 5 & 1ธ. $5-6$ & $2-2.2$ & $6-7$ & $12-12.5$ & $4.5 \mathrm{~m} 5$ & $1.75-2$ & $11-12$ \\
\hline Huernia sp.5 & $6 \mathrm{~mm} 8$ & $5.5-6$ & $1.5-2$ & $16-8$ & $7 \mathrm{~m} 01$ & $3.5-5$ & $1.5-2$ & $7-11$ \\
\hline
\end{tabular}

\begin{tabular}{|c|c|c|c|c|c|c|c|c|c|c|}
\hline \multirow{2}{*}{ se pceps } & \multicolumn{2}{|c|}{$\begin{array}{l}\text { Corolla- } \\
\text { lobes }\end{array}$} & \multirow{2}{*}{\begin{tabular}{|c|} 
Outer \\
corona \\
Diamete \\
$\mathrm{r}$ \\
$\mathrm{mm}$
\end{tabular}} & \multicolumn{2}{|c|}{ Inner corona } & \multicolumn{2}{|c|}{$\begin{array}{l}\text { ne ceeggca } \\
\mathrm{m}\end{array}$} & \multicolumn{2}{|l|}{ Corolla- } & \multirow[t]{2}{*}{$\begin{array}{c}\text { Intermediat } \\
\text { e lobes }\end{array}$} \\
\hline & el rr ea & \begin{tabular}{|c|} 
Widt \\
$\mathrm{h}$
\end{tabular} & & eael rr & $\begin{array}{c}\text { Widt } \\
\mathrm{h}\end{array}$ & $\begin{array}{c}\text { lengt } \\
\mathrm{h}\end{array}$ & Width & Length & Width & \\
\hline abica & $7-15$ & $17-22$ & $8.5-9$ & 4.8 & 1.1 & \begin{tabular}{c|}
$1.2-$ \\
1.3
\end{tabular} & $\begin{array}{c}2.4- \\
2.7\end{array}$ & $12-18$ & $8-15$ & $2--3.5$ \\
\hline H.kha & 7- 9 & $\mid 14-18$ & $4-5$ & 3.7 & 0.9 & $\begin{array}{c}0.2- \\
0.4\end{array}$ & $\begin{array}{c}1.1- \\
1.4\end{array}$ & $9-12$ & $12-13$ & 2.2 \\
\hline$p 1$ & $9-10$ & $15-16$ & $5.5-6$ & 3.6 & 1 & \begin{tabular}{|c|}
$0.3-$ \\
0.4
\end{tabular} & $\begin{array}{c}1.4- \\
1.5\end{array}$ & $11-12$ & $11-12$ & $1.5-1.75$ \\
\hline Huernia .sp. 2 & $11-12$ & $18-19$ & $6.5-7$ & 4.5 & 1.2 & $\begin{array}{c}0.5- \\
0.7\end{array}$ & $1.75-2$ & $\begin{array}{c}14.5- \\
15\end{array}$ & $14-15$ & $1.5-2$ \\
\hline $\begin{array}{l}\text { Huernia } \text { sp. } \\
\text { (Collenette 1176) }\end{array}$ & $11-14$ & $21-25$ & $8-10$ & 4.7 & 1.3 & $\begin{array}{c}0.8- \\
1\end{array}$ & $\begin{array}{c}1.7- \\
2.3\end{array}$ & $11-16$ & $9-15$ & $3.5--4.5$ \\
\hline Hue & $7-12$ & $\begin{array}{l}8- \\
14\end{array}$ & $5.75-6$ & 3.3 & 0.8 & $\begin{array}{c}0.2- \\
0.3\end{array}$ & $\begin{array}{c}1.3- \\
1.6\end{array}$ & $\begin{array}{c}05- \\
15\end{array}$ & $9-00$ & $1-1.25$ \\
\hline
\end{tabular}


Table 2. Qualitative morphological characters of the eight Huernia species under investigation.

\begin{tabular}{|c|c|c|c|c|c|c|c|c|c|}
\hline & \multirow{3}{*}{$\begin{array}{l}\text { Flower } \\
\text { odor }\end{array}$} & \multirow{3}{*}{$\begin{array}{l}\text { Corolla } \\
\text { color }\end{array}$} & \multirow{3}{*}{$\begin{array}{l}\text { Corolla- } \\
\text { lobes } \\
\text { Groove }\end{array}$} & \multirow{3}{*}{\begin{tabular}{|c|} 
Corolla- \\
lobes \\
Groove \\
spreading
\end{tabular}} & \multirow{3}{*}{\begin{tabular}{|c|} 
e ce eggam \\
Crcea
\end{tabular}} & \multirow{3}{*}{$\begin{array}{c}\text { Outer } \\
\text { corona } \\
\text { shape }\end{array}$} & \multicolumn{3}{|c|}{ Inner corona } \\
\hline s eaae & & & & & & & Base & shape & Tip shape \\
\hline & & & & & & & $\begin{array}{c}\text { From } \\
\text { bottom }\end{array}$ & \begin{tabular}{|c|}
$\begin{array}{c}\text { From dorsal } \\
\text { side }\end{array}$ \\
\end{tabular} & \\
\hline H.saudi arabica & $\begin{array}{c}\text { Unpleasant } \\
\text { smell }\end{array}$ & $\begin{array}{l}\text { Yellowish } \\
\text { creamy }\end{array}$ & Deep & Horizontal & aueeacg & $\begin{array}{l}\text { eClin m } \\
\text { e ur aC }\end{array}$ & huoed & $\begin{array}{c}\text { aueChoare } \\
\text { cr udamr am } \\
\text { r cCa }\end{array}$ & $\begin{array}{c}\text { Sub obtuse \& } \\
\text { Smooth }\end{array}$ \\
\hline H.khalidbinsultanii & $\begin{array}{c}\text { Very bad } \\
\text { smell }\end{array}$ & $\begin{array}{l}\text { Witch } \\
\text { creamy }\end{array}$ & Superficial & Ascending & mor anth & $\mathrm{eCk}$ & Triangular & \begin{tabular}{|c|} 
s n ugaem \\
aueChoarei \\
cr udam am \\
r cCa
\end{tabular} & $\begin{array}{c}\text { Obtuse \& } \\
\text { Tuberculation }\end{array}$ \\
\hline H.khalidbinsultanii 1 & $\begin{array}{c}\text { Very bad } \\
\text { smell }\end{array}$ & $\begin{array}{l}\text { Witch } \\
\text { creamy }\end{array}$ & Absent & Ascending & mor arsih & $\mathrm{eCk}$ & Straight & $\begin{array}{c}\text { s n uggem m } \\
\text { aueChoaree } \\
\text { cr udam am } \\
\text { r cCa }\end{array}$ & \begin{tabular}{|c|} 
Obtuse \& \\
Tuberculation
\end{tabular} \\
\hline H.khalidbinsultanii 2 & $\begin{array}{c}\text { Very bad } \\
\text { smell }\end{array}$ & $\begin{array}{l}\text { Witch } \\
\text { creamy }\end{array}$ & Deep & Ascending & mor anth & $\mathrm{eCk}$ & Triangular & Cnugge & \begin{tabular}{|c|} 
Obtuse \& \\
Tuberculation
\end{tabular} \\
\hline $\begin{array}{c}\text { Huernia sp. } \\
\text { (Collenette 1176) }\end{array}$ & $\begin{array}{c}\text { Unpleasant } \\
\text { smell }\end{array}$ & $\begin{array}{l}\text { Yellowish } \\
\text { creamy }\end{array}$ & Deep & Horizontal & aueeacg & e ur aC & s rhced r r & Cnugge & $\begin{array}{c}\text { Sub obtuse \& } \\
\text { Smooth }\end{array}$ \\
\hline Huernia sp.3 & $\begin{array}{c}\text { Very bad } \\
\text { smell }\end{array}$ & $\begin{array}{c}\text { Yellowish } \\
\text { creamy }\end{array}$ & Absent & Reflex & mor anth & $\mathrm{eCk}$ & Triangularr & $\begin{array}{c}\text { aueChoare } \\
\text { cr udamr am } \\
\text { r cCa }\end{array}$ & $\begin{array}{l}\text { Sub obtuse \& } \\
\text { Tuberculation }\end{array}$ \\
\hline Huernia sp.4 & $\begin{array}{c}\text { Unpleasant } \\
\text { smell }\end{array}$ & $\begin{array}{c}\text { Yellowish } \\
\text { brown }\end{array}$ & Superficial & Reflex & aueeacg & e ur aC & s rhed r r & convex & $\begin{array}{l}\text { Obtuse \& } \\
\text { Smooth }\end{array}$ \\
\hline Huernia sp.5 & $\begin{array}{c}\text { Very bad } \\
\text { smell }\end{array}$ & $\begin{array}{c}\text { Yellowish } \\
\text { brown }\end{array}$ & Deep & Reflex & aueeacg & e ur aC & s rhed r r & $\begin{array}{c}\text { aueChoare } \\
\text { cr udamr am } \\
\text { r cCa }\end{array}$ & $\begin{array}{c}\text { Sub obtuse \& } \\
\text { Smooth }\end{array}$ \\
\hline
\end{tabular}

From the results of all the morphological features of the studied material, the Huernia spesies can be separated into eight different taxa including identified species e.g.: $H$. saudi-arabica, $H$. khalidbinsultanii, $H$. sp. 1, H. sp. 2, Huernia sp. (collenette1176), Huernia sp.3, Huernia sp.4, and Huernia sp.5.

These examined characters divided the eight specimens into two groups: The first group is distinguished by the hairy-like papillae covering the corolla's inner surface. This group includes H. khalidbinsultanii (Figure 3), $H$. sp. 1 (Figure 4), H. sp. 2 (Figure 5), and H. sp.5 (Figure 9). This group is 
further divided into two subgroups based on the corolla base color and spreading lobes: the first subgroup includes only Huernia sp.5 (Figure 7b), which is characterized by a yellow creamy corolla color with reflexed lobes; the second subgroup has white creamy corolla color with steeply ascending lobes and this includes the three remaining species of the $H$. khalidbinsultanii, $H$. sp. 2 (Figure 5 b) which characterized by large flowers more than $3.5 \mathrm{~cm}$ in diameter and long pedicels $(>10 \mathrm{~mm}$ ), whereas $H$. khalidbinsultanii (Figure 3b) and H.sp. 1 (Figure 4b ) with small flowers (less than $3.5 \mathrm{~cm}$ diameter) and short pedicels $(<10 \mathrm{~mm})$. These two species can be differentiated by the triangular inner corona base in $H$. khalidbinsultanii (Figure 3c) and the straight base in $H$. sp. 1 (Figure 4c).

The second group includes $H$. saudi-arabica (Figure 2), Huernia sp. (collenette1176) (Figure 6), Huernia sp.3 (Figure 7), and Huernia sp.4 (Figure 8), which were characterized by the conical papillae covering the corolla's inner surface. This group was further divided into two subgroups based on the multiplying of stem angles: The first subgroup can be distinguished by the five-angled stems, which multiply to 6-8 angles in the tip of Huernia sp.5 stems (Figure 9a), whereas the second subgroup includes Huernia sp. (collenette1176) (Figure 6a), Huernia sp.4 (Figure 8a) and $H$. saudi-arabica (Figure 2a), which are characterized by five-angled stems without multiplication. The second subgroup was divided into two subgroups based on the width of the papillae base: $H$. saudi-arabica was distinguished by broad base papillae (Figure 2c); both Huernia sp. (collenette1176) (Figure 6c) and Huernia sp.4 (Figure 8c) were characterized by narrow base papillae. Furthermore, Huernia sp.4 was distinguished by a broad corolla tube and short intermediate lobes (Figure 8f), whereas Huernia sp. (collenette1176) (Figure 6f) was distinguished by a narrow corolla tube and long intermediate lobes.

The study of similarity matrix based on morphological data (Table 3) showed that the closest relationship among the species under investigation was $65 \%$, between the two species $H$. khalidbinsultanii and $H$. sp. 1 , followed

by the relationship (45\%) between the two species $H$. sp. 1 and $H$. sp 2; finally, the relationship (46\%) between $H$. khalidbinsultanii and $H$. sp 2, a result that supports the proposal to treat $H$. khalidbinsultanii , $H$. sp. 1 and $H$. sp 2 as a different species in one complex.

The table also shows that the lowest value of the similarity among the species was between species Huernia sp.3 and Huernia sp.4 (3\%), followed 
by $H$. khalidbinsultanii and Huernia sp. (collenette1176) (7\%) from the rest of the species.

Table 3. Similarity matrix based on 26 morphological characters for 8 Huernia species under investigation

\begin{tabular}{|c|c|c|c|c|c|c|c|c|}
\hline Samples & 1 & 2 & 3 & 4 & 5 & 6 & 7 & 8 \\
\hline 1 & 1 & 0.23 & 0.26 & 0.26 & 0.50 & 0.23 & 0.34 & 0.38 \\
\hline 2 & & 1 & 0.65 & 0.46 & 0.07 & 0.50 & 0.19 & 0.19 \\
\hline 3 & & & 1 & 0.45 & 0.11 & 0.50 & 0.11 & 0.23 \\
\hline 4 & & & & 1 & 0.19 & 0.30 & 0.34 & 0.15 \\
\hline 5 & & & & & 1 & 0.23 & 0.42 & 0.42 \\
\hline 6 & & & & & & 1 & 0.03 & 0.23 \\
\hline 7 & & & & & & & 1 & 0.30 \\
\hline 8 & & & & & & & & 1 \\
\hline
\end{tabular}

(1: H. saudi arabica; 2: H. khalidbinsultanii ; 3: $H$.sp. $1 ; 4: H$ sp. 2 ; 5: Huernia sp.(collenette1176) ; 6:Huernia sp.3 ; 7 : Huernia sp. 4 ; 8: Huernia sp. 5).
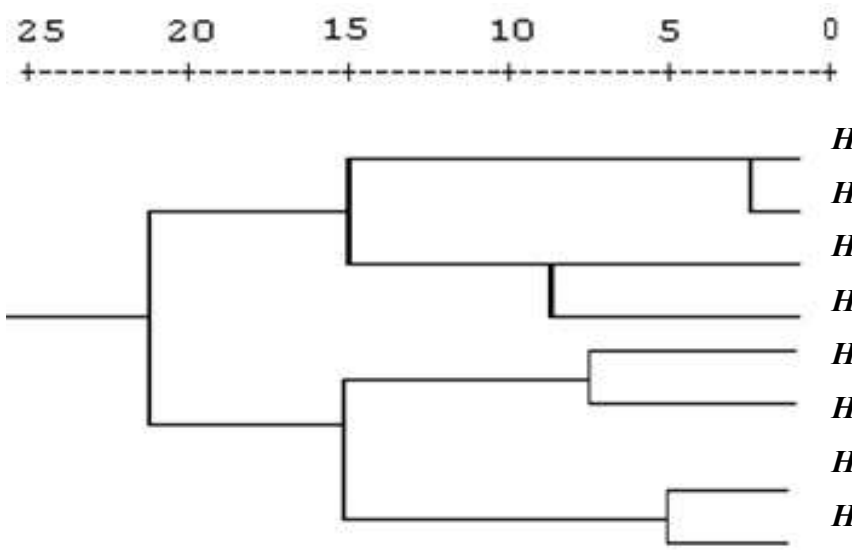

Huernia sp. 4

Huernia sp. 2

Huernia. sp. 1

H. khalidbinsultanii

H. saudi arabica

Huernia sp .5

Huernia sp. (collenette1176)

Huernia sp. 3

Fig. 1. Dendogram showing the relationship between the eight Huernia sp. under investigation.

From the results of the similarity matrix, an UPGMA dendrogram (Figure 1) was constructed as part of investigations into dissimilarity 
computed among Huernia species. The eight Huernia species were grouped into two main clusters: the first cluster included subsections with four species where $H$. sp. 1 and $H$. khalidbinsultanii are closely allied, with $H$. sp. 2. The second cluster included subsection with the other four species allied to those species pair. $H$. sp. 3 and $H$. sp.5 were different from all the others. From the above results, the delimitation of Huernia species needs more investigations

\section{Morphological Key-}

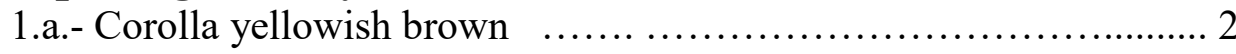

1.b. Corolla not yellowish brown ................................ 3

2.a. Five angled stem multiplying to $6-8$ at tip .................. Huernia. sp.5

2.b. Stem 5-angled without multiplication ................. Huernia.sp. 4

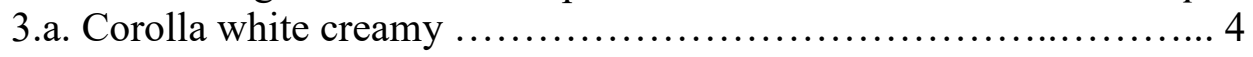

3.b. Corolla yellowish creamy .................................... 6

4.a. Diameter of outer corona 4-5 $\mathrm{mm}$.................. H.khalidbinsultanii

4.b. Diameter of outer corona $5.5-7 \mathrm{~mm}$.......................... 5

5.a. length of stem tubercles $10-14 \mathrm{~mm}$..................... Huernia. sp. 1

5.b. Length of stem tubercles less than $10 \mathrm{~mm}$ long .......... Huernia. sp. 2

6.a. Base of inner corona swollen ............. Huernia sp.(collenette1176)

6.b. Base of outer corona constructing above the base .................. 7

7.a. Papillae of inner corona over $1 \mathrm{~mm}$ long .................H. saudi arabica

7.b. Papillae of inner corona less than $1 \mathrm{~mm}$ long ............. Huernia.sp. 3 

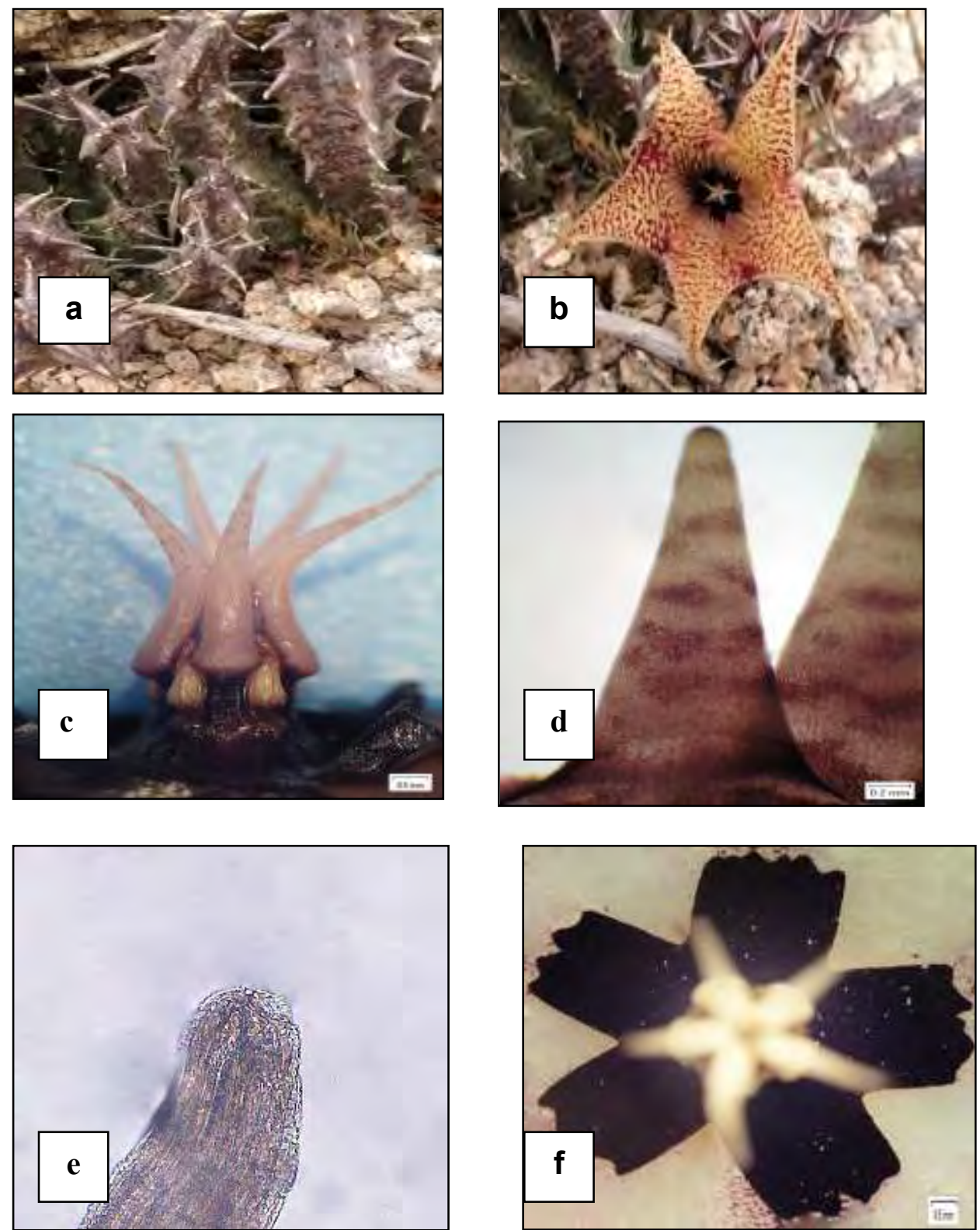

Fig. 2. Huernia saudi arabica a) 5angled stem b) Yellowish creamy flower deep corolla lobes grove, c) Gynostegium and round base eeealmm corona d) Conical Papillae e) Sub obtuse tip of inner corona f) Outer corona disk and lobes shape. 

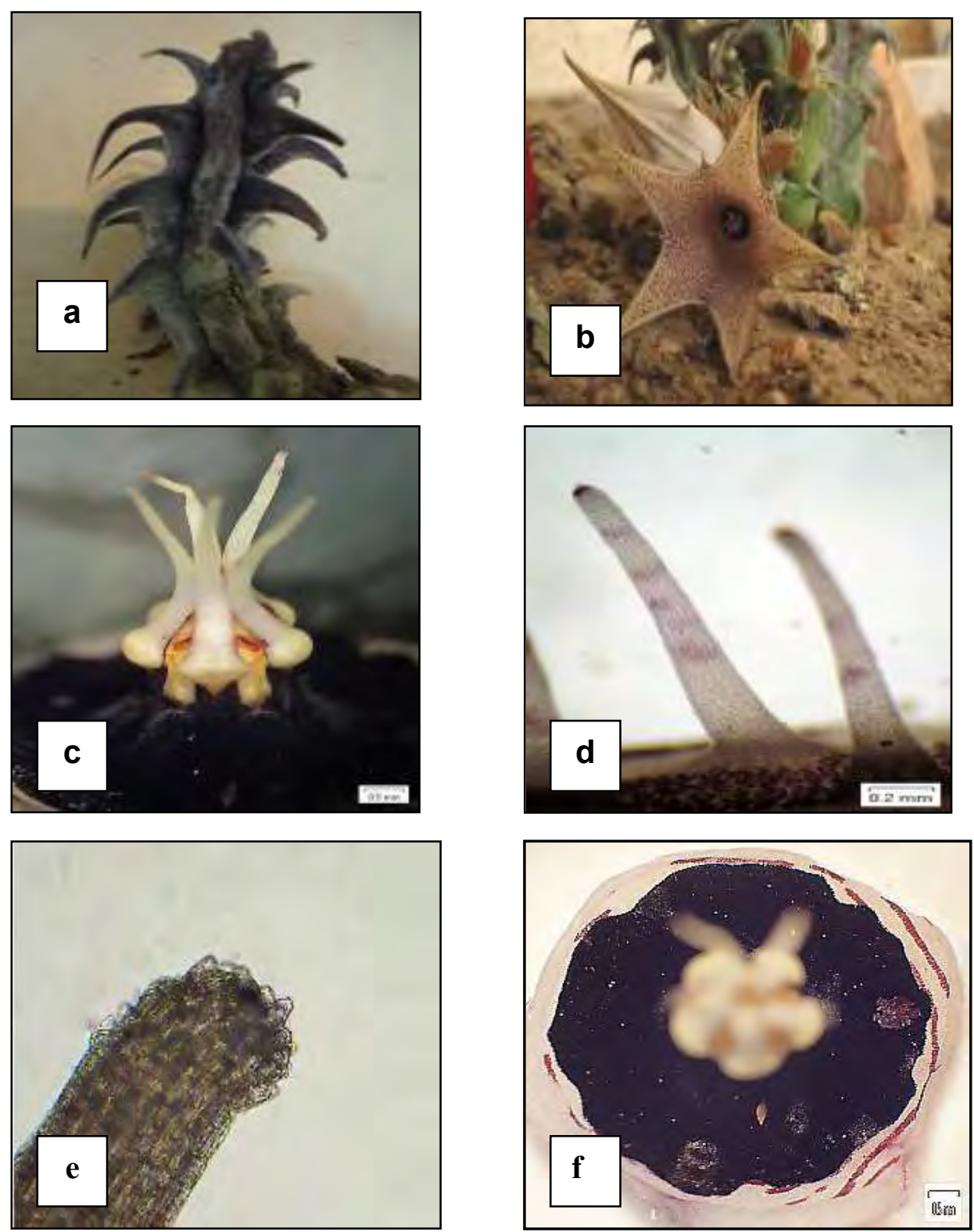

Fig. 3. Huernia khalidbinsultanii a) 5angled stem b) Witch creamy flower superficial corolla lobes groove c) Gynostegium and triangular $\mathrm{mm}$ neeahcoronand) Tube form papillae e) Obtuse tuberculation tip of inner corona f) Outer corona disk shape. 
Taxonomic Studies on some species of genus Huernia R.Br. ......
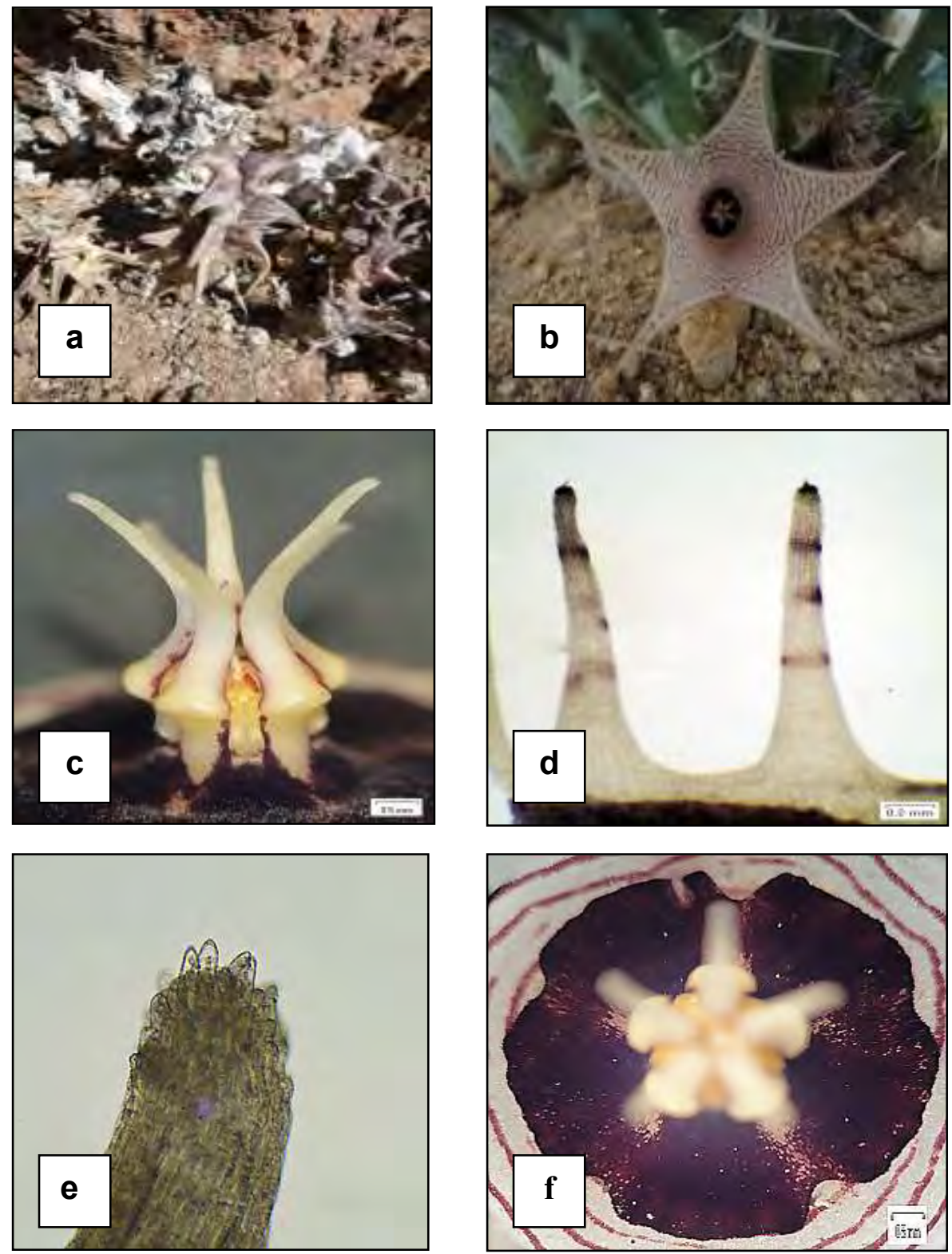

Fig. 4. Huernia khalidbinsultanii 1 a) 5angled stem b) witch creamy flower no corolla lobes groove, c) Gynostegium and straight eeeahmm corona d) Tube form papillae e) Obtuse tuberculation tip of inner corona f) Outer corona disk shape. 

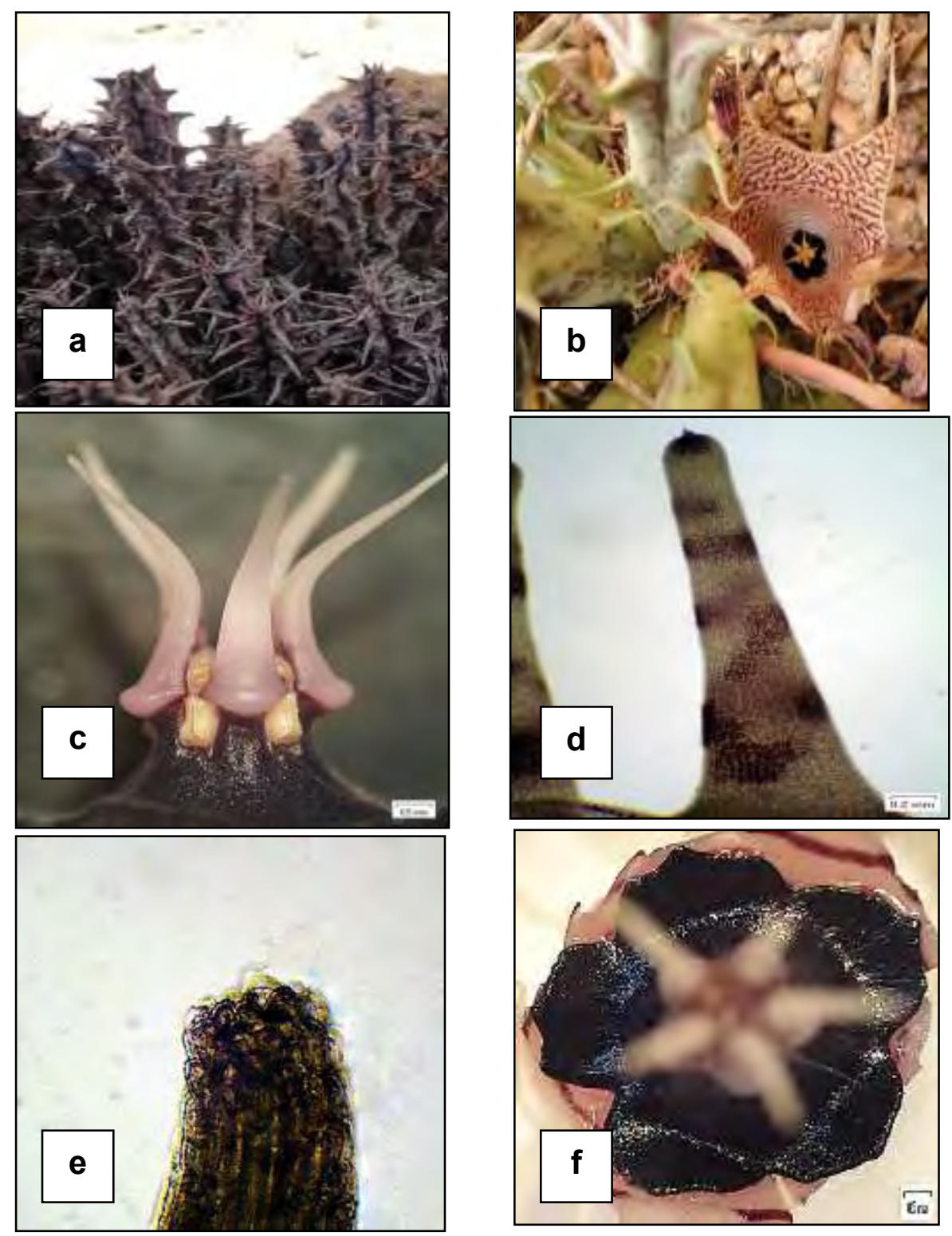

Fig. 5. Huernia khalidbinsultanii 2 a) 5angled stem b) Witch creamy flower superficial corolla lobes groove, c) Gynostegium and triangular reeeahmoronand)Tube form papillae e) Obtuse tuberculation tip of inner corona f) Outer corona disk shape. 

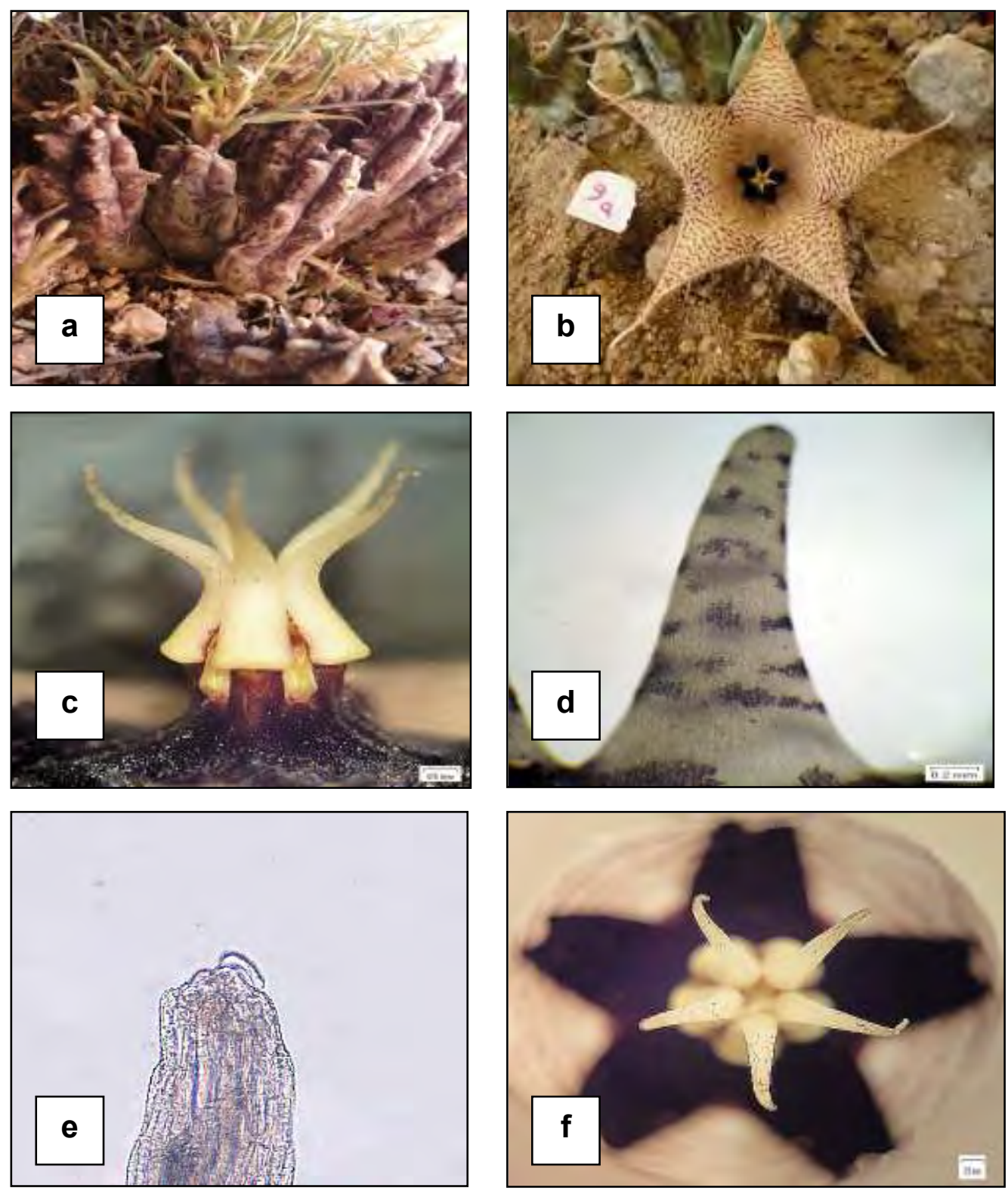

Fig. 6. Huernia sp. (collenette 1176 ) a) 5angled stem, b) Yellowish creamy flower deep corolla lobes grove, c) Gynostegium and straight reeahmonand)Conical papillae e) Sub obtuse smooth tip of inner corona f) Outer corona Lobes shape. 

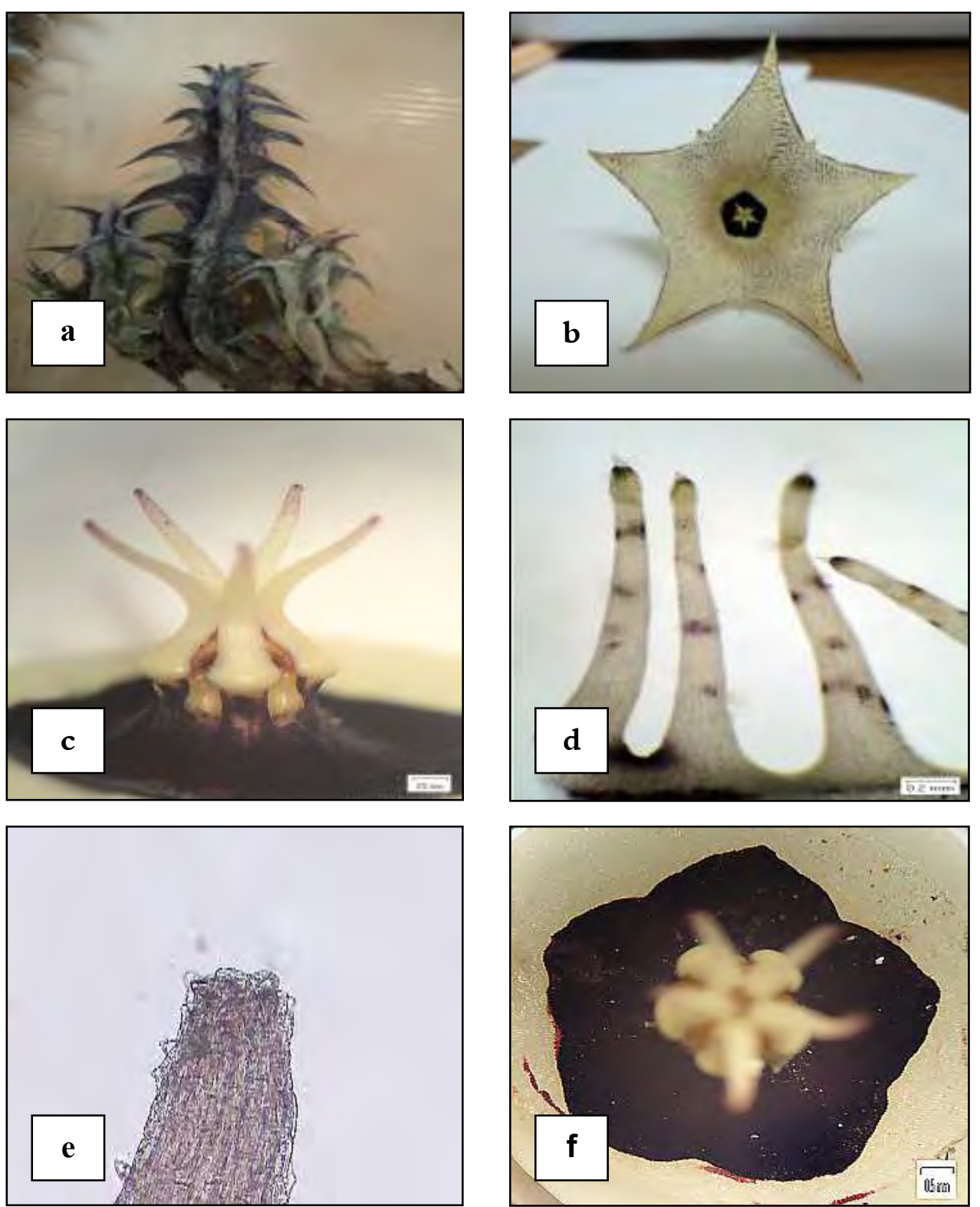

Fig. 7. Huernia sp. 3 a) 5angled stem , b) Yellowish creamy flower absent of corolla lobes groove, c) Gynostegium and triangular empi corona, d) Tube form papillae e) Sub obtuse tuberculation tip of inner corona f) Outer corona disk shape 

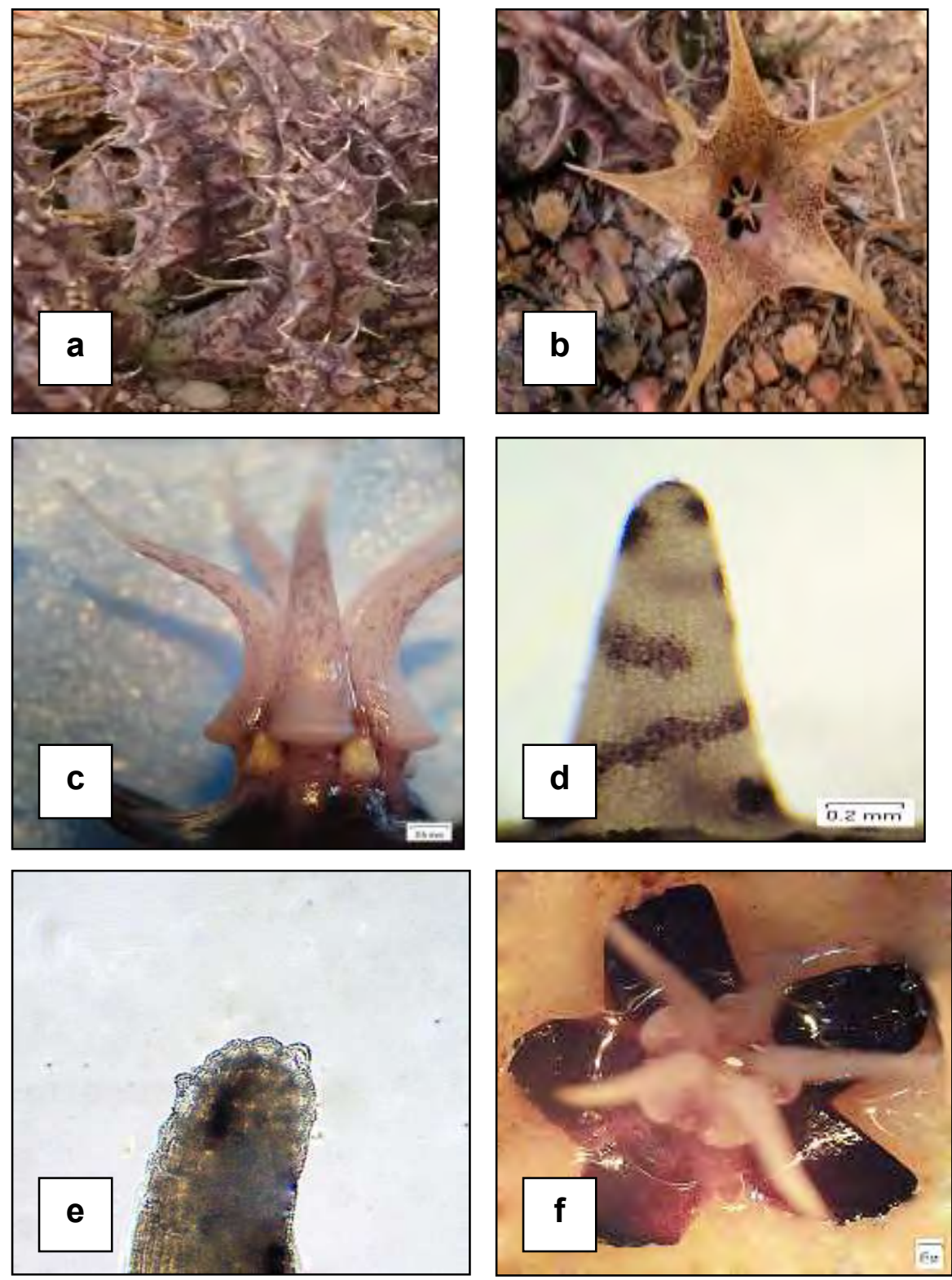

Fig. 8. Huernia sp. 4 a) 5angled stem b) Yellowish brown flower with superficial corolla lobes groove, c) Gynostegium and straight reeealmm coronard)Conical papillae e) Obtuse and smooth tip of inner corona f) Outer corona lobes shape. 

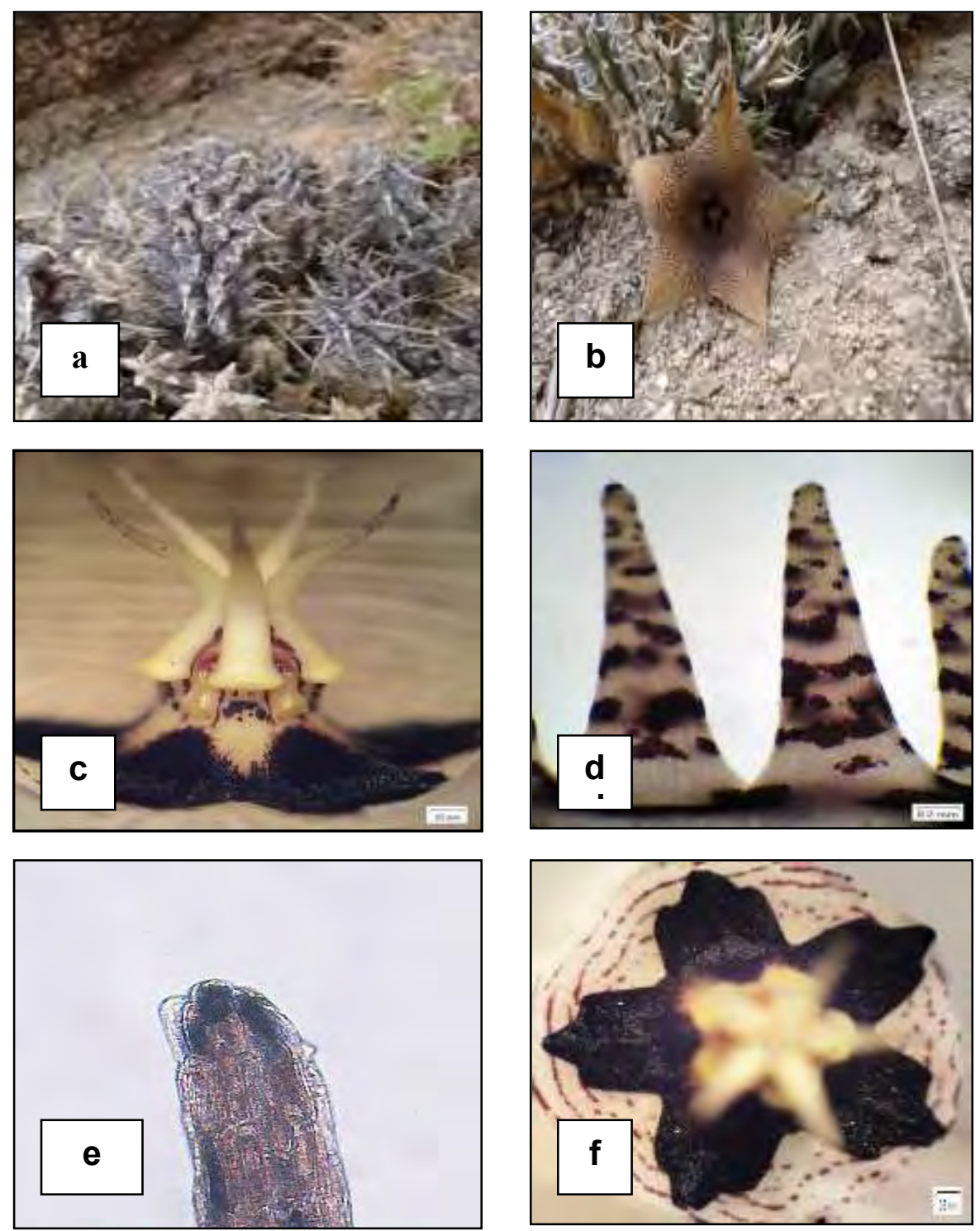

Fig. 9. Huernia sp. 5 a) 6-8 angled stem, b Yellowish brown flower with deep corolla lobes groove, c) Gynostegium and straight reeealmm coronamd) Conical papillae e) Sub obtuse smooth tip of inner corona f) Outer corona lobes shape 


\section{References}

Albert, F., \& Meve, U. (2002). Illustrated handbook of succulent plants:Asclepiadaceae. Berlin: Springer.

Al-Hemaid, F. M. (2001). A New Species of Huemia R. Br. (Asclepiadaceae) From Saudi Arabia. Saudi. J. Biol. Sci., Vol. 8, No. 2 , pp. 166-170 .

Armstrong, W.P.(1997). Carrion flowers: Not all flowers smell as sweet as a Rose. ZOONOOZ 70 (1): 24-27

Goyder, D.J.\& Al-Hemaid, F.M.A.(2009).The identity of the Saudi Arabian species Huernia haddaica Hemaid. Asklepios 106: 27 - 28.

Brown, N. E. 1895. Diagnoses Africanae 8. Bull. Misc. In! Kew p.265

Bruyns, P. (2005). Stapeliads of Southern Africa and Madagascar. Hatfield, South Africa: Umdaus Press.

Chaudhary, S. A. and AI-Jowaid, A. 1999. Vegetation of the Kingdom of Saudi Arabia. Ministry of Agriculture, Riyadh.

Chaudhary, S. A. (2001). Flora of the kingdome of Saudi Arabia. Illustrated vol.2 (part 2). Kingdom of Saudi Arabia.: Ministry of Agriculture and Water.

Collenette, S. (1985). An Illustrated Guide to the Flowers of Saudi Arabia. London.: Scorpion Publishing.

Collenette, S. (1998). Check Iist of botanical species in Saudi Arabia. U.K: International Asclepiad Society.

Collenette, S. (1999). Wildflowers of Saudi Arabia. Riyadh: CWCD.

Court, D. (2000). Succulent Flora of Southern Africa (Vol. revised ed.). Netherlands: A.A.BALKEMA.

Field, D. (1980). A new Huernia (Asclepiadaceae) from Saudi Arabia, with notes on related species. Kew Bull vol.35 (4) , pp. 753-757.

Forsskal, P. 1975. Flora of Acgyptica-Arabica. Huernia

Heemstra, Al Hassan and Al Minwer (1990). Plants of Northern Saudi Arabia, pp. 357.

ICBN International Code of Botanical Nomenclature (Vienna Code), Regnum Vegetabile 2006, 146: 1 - 568.

Lavranos, J.J. (1963). Two new species of Huernia from south-western Arabia. Journ. S.Afr. Bot. 29:97-101

Leach, L. (1976). A preliminary review of the prominently papillose Huernia species (Asclepiadaceae). J. S. Afr. Bot. 42 (4), pp. 439487. 
Leach, L.C. (1995) The preparation of good (useful) herbarium specimens of succulent plants especially of the spiny ephorbias and of the stapeliads and aloes, Asklepios ,64: pp. 24-26.

Mighahid, A. (1996). Flora of Saudi Arabia. Riyadh: King Saud University. Miller, A. G. and Cope, T.A. (1996) Flora of the Arabian Peninsula, Vol I. Riyadh

Plowes DCH, McCoy TA. (2003). Huernia khalidbinsultanii, a new species from the Kingdom of Saudi Arabia. Cact. Succ. J. (USA) 75. (1): 1921

Rowley, G. (2004). Huernia R.Brown. In The European Garden Flora :A Manual for the Identification of Plants Cultivated in Europe, Both Out-of-Doors and under Glass Vol.6 Dicotyledons( part 4) :Loganiaceae to Compositae. cambridge: cambridge university press.

Schwrtz, O. (1939). Flora des tropischen Arabien, P.195, Hamburg.

Thomas, J. (2010 a ) Endemic plants of Saudi Arabia, Research Projects Biodiversity study of selected wetland ecosystems of Saudi Arabia, King Saud University .

Thomas, J. (2010 b) Flora of Saudi Arabia list of species. Research Projects Biodiversity study of selected wetland ecosystems of Saudi Arabia, King Saud University .

Wood, 1. R. I. (1997). A handbook of the Yemen Flora, Whitstable Litho Printers Ltd., UK 